\title{
Éditorial : L'avenir de la formation en gérontologie au Canada et autres notes distinctes
}

Dans mon dernier éditorial du CJA/RCV (volume 28, numéro 1, 2009), je suggérais que nous pourrions devoir ajuster notre façon de penser en raison de la présente récession et de la manière dont elle affecte actullement les Canadiens âgés et la société canadienne en général et don't elle les affectera dans l'avenir. Cependant, je ne pensais pas écrire si tôt au sujet de l'influence des conditions économiques actuelles sur nos universités et collèges et du rôle de la formation de premier cycle en gérontologie.

$\mathrm{Au}$ cours des 12 derniers mois, nous avons entendu parler d'une éventuelle réorganisation des programmes de premier cycle en gérontologie dans les universités canadiennes. Mon objectif n'est pas de commenter les détails entourant les raisons et les décisions. Je ne suis pas en mesure de porter un jugement sur les universités, sur toute personne associée à celles-ci, ou sur les raisons sous-jacentes justifiant les décisions prises, ou qui seront prises, concernant le rôle de la formation de premier cycle en gérontologie, dans des universités spécifiques, à l'avenir.

Par contre, je serais surpris d'entendre un quelconque dirigeant d'université mettre en doute que : la société canadienne et bon nombre de sociétés à travers le monde sont vieillissantes; qu'entre 2025 et 2050, le quart de la population du Canada aura 65 ans et plus; et que cette réalité exigera la formation d'une maind'œuvre spécialisée, ainsi que la préparation non formelle de la population, afin qu'elle puisse venir en aide aux personnes âgées lorsqu'elles ne pourront plus vivre de manière autonome. L'élément déconcertant est que si nos dirigeants universitaires partagent la même perspective que la nôtre, alors pourquoi ont-ils tendance à couper dans les programmes de gérontologie en ces temps difficiles? La formation en gérontologie ne devrait-elle pas devenir une priorité ? $\mathrm{Ne}$ devrions-nous pas mieux faire valoir notre cause non seulement pour la préservation des programmes de formation existants, mais aussi pour leur expansion?
Quelle est la place de la formation en gérontologie dans nos universités ? Devrions-nous donner la formation au premier ou au deuxième cycle ? Il est difficile de répondre à ces questions, mais je suis convaincu qu'elles doivent faire l'objet de discussions et de débats dans le CJA/RCV ainsi que lors de nos réunions annuelles.

En ce qui concerne un partenariat qui remonte au temps où l'Institut du vieillissement -Instituts de recherche en santé du Canada (IV - IRSC) ne faisait qu'amorcer ses activités et que le CJA/RCV transigeait vers son format actuel, je suis fier de vous annoncer que l'IV - IRSC a conclu une entente permettant la continuité de ce partenariat. À partir du présent numéro, le CJA/RCV prévoit publier une nouvelle section, intitulée : Institut du vieillissement - Instituts de recherche en santé $d u$ Canada : Profils. Profils apparaîtra normalement dans les deuxième et quatrième numéros de chaque année et mettra l'accent sur les préoccupations qui ressortent des recherches subventionnées par l'IV - IRSC.

Pour conclure, je vous invite à vous rappeler de l'article que j'ai écrit au sujet de rendre le journal davantage écologique, dans le CJA/RCV volume 27, numéro 2, 2008. Lorsque nous nous sommes joints à la Cambridge University Press, nous avons décidé d'utiliser un papier différent, conforme aux normes établies par le Forest Stewardship Council (FSC) http://www.fsc.org/ (site en français, version Canada : http://www.fsccanada.org / francais.htm). Grâce à cette approche, le CJA/ $\mathrm{RCV}$ fera sa part dans la préservation des forêts à travers le monde.

Comme à l'habitude, n'hésitez pas à me contacter à l'adresse mark.rosenberg@queensu.ca si vous avez des commentaires à propos de cet éditorial ou de tout autre sujet du CJA/RCV.

Mark Rosenberg

Rédacteur en chef 